\title{
On The Operation Configuration of SiGe HBTs Based on Power Gain Analysis
}

\author{
Zhenqiang Ma, Member, IEEE, and Ningyue Jiang, Student Member, IEEE
}

\begin{abstract}
The power gain difference, under different device stability conditions, between common-emitter (CE) and common-base $(\mathrm{CB})$ bipolar junction transistors $(\mathrm{BJT})$ is analyzed comprehensively. The analysis reveal that the $\mathrm{CB}$ configuration offers higher maximum available power gain than the $\mathrm{CE}$ configuration in the device's high operation frequency range, while the inverse relation holds in the very low frequency range. In the intermediate frequency range, the base resistance value, mainly affected by the base doping concentration, determines which configuration offers higher maximum stable power gain (MSG). These analyses have explicit implications on the operation configurations of SiGe heterojunction bipolar transistors (HBTs). Employing a typical doping profile of $\mathrm{Si}$ bipolar junction transistors with a trapezoidal Ge profile in SiGe HBTs usually results in a larger base resistance than the emitter resistance. For these devices, the CE configuration exhibits higher MSG than the CB configuration. Employing a higher base doping concentration than the emitter with a box-type Ge profile considerably reduces the base resistance and thus favors the CB configuration for power amplification in this frequency range. The analysis are quantitatively verified with simulation and measurement results from SiGe HBTs of representative $\mathrm{Ge}$ and base doping profiles.
\end{abstract}

Index Terms-Common-base (CB), common-emitter (CE), doping profile, maximum available power gain (MAG), maximum stable power gain (MSG), power gain, SiGe heterojunction bipolar transistors (HBTs), two-dimensional (2-D) simulation.

\section{INTRODUCTION}

$\mathbf{S}$ iGe-based power heterojunction bipolar transistors (HBTs) have recently emerged as competitive alternatives to III-V devices for RF and microwave power amplification [1]-[17]. While superior RF performance has been demonstrated, through vertical and lateral scaling, on low power, high-speed SiGe HBTs [18], [19], the heterostructure design of SiGe HBTs for high power amplification, including region thickness, doping profile and Ge profile, differs from that for low-power and high-speed applications. Most SiGe HBTs integrated in SiGe BiCMOS adopt the typical doping profile of silicon bipolar junction transistors (BJT) (the high-to-low doping concentration declines from emitter to base and then to collector region) with a trapezoidal Ge profile. For high power amplification, these devices are customized to offer high breakdown voltage

Manuscript received July 21, 2004; revised November 29, 2004. This work was supported in part by the NSF under Grant ECS 0323717 and in part by the University of Wisconsin, Madison under the Start-Up Fund. The review of this paper was arranged by Editor J. N. Burghartz.

The authors are with the Department of Electrical and Computer Engineering, University of Wisconsin, Madison, WI 53706 USA (e-mail: mazq@engr.wisc.edu).

Digital Object Identifier 10.1109/TED.2004.842541 characteristics by lowering their collector doping concentrations [16]. Despite the prevalence of this typical doping profile and Ge profile, a number of SiGe power HBTs [1], [2], [9]-[11], [13] employing a heavily doped base region (higher than the emitter region, typical of III-V HBTs) have been demonstrated, by taking the advantage of having a large valence band offset in presence of a box-type Ge profile. No matter which type of Ge profile and doping profile are employed for SiGe HBTs, power gain values are of the most important concern for designers when employing these devices for power amplification. A higher power gain can reduce the required input RF power level, reduce the number of power amplification stages of a power amplifier module, improve power added efficiency (PAE $\left.=(G-1) \bullet P_{\mathrm{in}} / P_{\mathrm{DC}}\right)$ and, thus, reduce the amount of heat generation within the power devices. In order to obtain the maximum power gain values, both common-emitter (CE) [2], [4], [8], [12], [16] and common-base (CB) [1], [9]-[11], [13] configurations have been attempted for power amplification using SiGe HBTs. However, to date, a recognized fundamental explanation on which configurations ought to be judiciously employed for power amplification has not been available.

On the other hand, although a heavily doped base region is prevailingly employed for III-V HBTs due to the pronounced heterojunctions at the emitter-base junctions, it is shown that different RF characteristics are still exhibited between the CE and the CB configurations [20]-[22]. A preliminary study on the difference of power gain characteristics between the two configurations of AlGaAs-InGaAs HBTs was done by Tanaka et al. [23]. In their study, the superior power gain characteristics using the CB configuration for III-V HBTs in a certain frequency range were recognized. However, a generalized power gain relationship between the two configurations that can be applied to BJTs of any doping profile and to the entire frequency range of interest was not given. For example, the derived relation of power gain between the two configurations on the III-V HBTs cannot be applied to SiGe HBTs having a typical Si BJT doping profile, nor to any bipolar transistors having a value of $f_{T}$ much larger than that of $f_{\max }$. Moreover, the frequency points where the power gain values of the two configurations converge, which can guide the HBT design and the selection of operation configurations, was not provided. It is thus necessary to provide an in-depth study on the general difference of power gain characteristics between the $\mathrm{CE}$ and $\mathrm{CB}$ configurations in order to clarify which configuration for SiGe HBTs (and other BJTs) should be used for power amplification in different frequency ranges. The study can also provide valuable design guidelines for SiGe power HBTs in order to maximize their power gain values. 


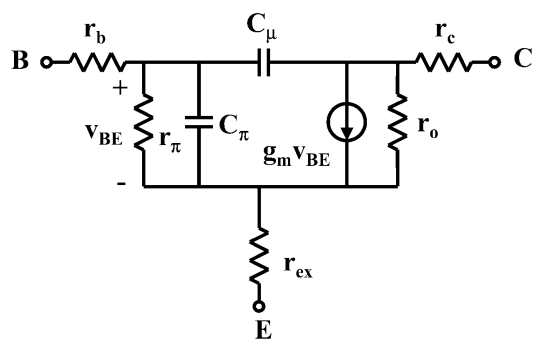

(a)

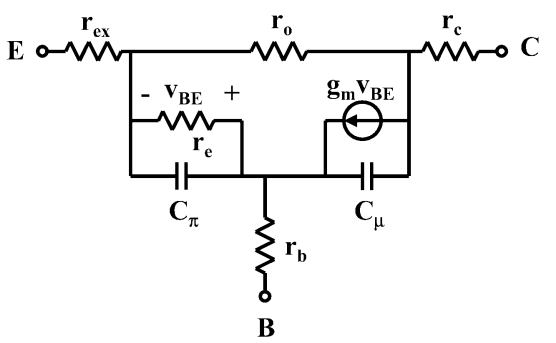

(b)

Fig. 1. (a) Small-signal hybrid- $\pi$ model for CE SiGe HBTs. (b) Small-signal $T$ model for CB SiGe HBTs.

In this paper, we first derive the power gain $G_{\max }$ from the small-signal equivalent circuits of the CE and the CB bipolar transistors and discuss the different behaviors of $G_{\max }$ in particular frequency ranges under different device stability conditions. We then analyze the implications of the frequencyand configuration-dependent power gain characteristics of SiGe HBTs having different doping and Ge profiles. Two-dimensional (2-D) simulations and measurement results are used to quantitatively verify our analysis.

\section{Small-Signal Power Gain in CE AND CB CONFIGURATIONS}

Although the Mason's unilateral power gain $(U)$ has been generally extrapolated to find the maximum oscillation frequency $\left(f_{\max }\right)$ of bipolar transistors, it shows no differences among different configurations (as seen in [22]) as it is defined. Therefore, this figure of merit does not signify the actual power amplification capability of BJTs or HBTs configured differently (It is useful to calculate $f_{\max }$, as $U$ and $G_{\max }$ converge to the same $f_{\max }$ [21], [22]). The absence of stability information in $U$ also makes it a deficient figure of merit for circuit implementations. Consequently, it is imperative to examine the power gain, $G_{\max }$ (maximum available gain/maximum stable gain, MAG/MSG) of bipolar transistors and to compare their differences between the $\mathrm{CE}$ and the $\mathrm{CB}$ configurations.

The small-signal hybrid- $\pi$ model equivalent circuit of $\mathrm{SiGe}$ HBTs, for the CE configuration, and the small-signal $T$-model equivalent circuit, for the $\mathrm{CB}$ configuration, which are equivalent to each other [24], are shown in Fig. 1. The parasitic emitter resistance $\left(r_{\mathrm{ex}}\right)$ and collector resistance $\left(r_{c}\right)$ are included in these two equivalent circuits for completeness of analysis. These two resistances were usually ignored in the equivalent circuits of Si BJTs [24] due to their negligible values in comparison to total base resistance $\left(r_{b}\right)$. They were also ignored in the simplified equivalent circuits of SiGe HBTs that have low base doping concentrations (lower than the emitter doping concentration) for the same reason. However, for SiGe HBTs that have a much higher doping concentration in the base region than in the emitter region, $r_{b}$ can be reduced to a much smaller value and becomes comparable to $r_{\mathrm{ex}}$ and $r_{c}$. As a result, both $r_{\mathrm{ex}}$ and $r_{c}$ have to be included in the equivalent circuits in this case.

The $H$-parameters of the two-port network representing the CE configuration small-signal equivalent circuit [Fig. 1(a)] are derived as the following (subscript symbols: $\mathbf{i}$, input port; $\mathbf{o}$, output port; $\mathbf{r}$, reverse transmission; $\mathbf{f}$, forward transmission; e, common-emitter; and b, common-base)

$$
\begin{aligned}
& h_{i e}=r_{b}+\frac{\left(\frac{1}{j \omega C_{\mu}}+r_{c}\right)\left[Z_{1}+r_{\mathrm{ex}}\left(1+g_{m} Z_{1}\right)\right]}{\frac{1}{j \omega C_{\mu}}+Z_{1}+\left(r_{\mathrm{ex}}+r_{c}\right)\left(1+g_{m} Z_{1}\right)} \\
& h_{r e}=\frac{r_{\mathrm{ex}}\left(1+g_{m} Z_{1}\right)+Z_{1}}{\frac{1}{j \omega C_{\mu}}+Z_{1}+\left(r_{\mathrm{ex}}+r_{c}\right)\left(1+g_{m} Z_{1}\right)} \\
& h_{f e}=\frac{\frac{g_{m} Z_{1}}{j \omega C_{\mu}}-Z_{1}-r_{\mathrm{ex}}\left(1+g_{m} Z_{1}\right)}{\frac{1}{j \omega C_{\mu}}+Z_{1}+\left(r_{\mathrm{ex}}+r_{c}\right)\left(1+g_{m} Z_{1}\right)} \\
& h_{o e}=\frac{1+g_{m} Z_{1}}{\frac{1}{j \omega C_{\mu}}+Z_{1}+\left(r_{\mathrm{ex}}+r_{c}\right)\left(1+g_{m} Z_{1}\right)}+\frac{1}{r_{o}}
\end{aligned}
$$

where $Z_{1}$ is given by $Z_{1}=r_{\pi} /\left(1+j \omega r_{\pi} C_{\pi}\right)$ and $r_{\pi}=\beta / g_{m}$ [24], $g_{m}=q I_{c} / k T(k T=26 \mathrm{meV})$. These four $H$-parameters, $h_{i e}, h_{r e}, h_{f e}$ and $h_{o e}$ are equivalent to $h_{11}, h_{12}, h_{21}$ and $h_{22}$ in the general two-port network format, respectively. Similarly, the $H$-parameters for the $\mathrm{CB}$ configuration equivalent circuit can be derived as

$$
\begin{aligned}
h_{i b} & =\frac{r_{b}\left(1-g_{m} Z_{2}+j \omega r_{c} C_{\mu}\right)}{1+j \omega C_{\mu}\left(r_{b}+r_{c}\right)}+r_{\mathrm{ex}}+Z_{2} \\
h_{r b} & =\frac{r_{b}\left(\frac{1-g_{m} Z_{2}}{Z_{2}+r_{o}}+j \omega C_{\mu}\right)+\frac{Z_{2}}{Z_{2}+r_{o}}}{1+\left(\frac{1-g_{m} Z_{2}}{Z_{2}+r_{o}}+j \omega C_{\mu}\right)\left(r_{b}+r_{c}\right)} \\
h_{f b} & =-\frac{g_{m} Z_{2}+j \omega r_{b} C_{\mu}}{1+j \omega C_{\mu}\left(r_{b}+r_{c}\right)} \\
h_{o b} & =\frac{1}{r_{b}+r_{c}+\frac{1}{j \omega C_{\mu}+\frac{1-g_{m} Z_{2}}{Z_{2}+r_{o}}}}
\end{aligned}
$$

where $Z_{2}$ is given by $Z_{2}=r_{e} /\left(1+j \omega r_{e} C_{\pi}\right)$ and $r_{e}=1 /\left(g_{m}+\left(1 / r_{\pi}\right)\right)=\beta /\left(g_{m}(1+\beta)\right)=\alpha / g_{m}$ [24]. In order to calculate the difference/ratio of power gain between two configurations, we make reasonable approximations (justified by actual values) to simplify the derived $H$-parameters and the power gain expressions in different frequency ranges.

\section{A. Intermediate-Frequency Range}

Since most of the SiGe HBTs are operated in the intermediate frequency range within the $f_{\max }$ of the devices (for RF and microwave power amplification) and the devices are potentially unstable in this frequency range [25], it is imperative to specifically consider the maximum stable power gain (MSG) in 
this useful frequency range. MSG can be expressed in terms of $H$-parameters as shown in (9)

$$
\mathrm{MSG}=\left|\frac{h_{f}}{h_{r}}\right| .
$$

From (2) and (3), the maximum stable gain MSG for the CE configuration can be derived as the following:

$$
\begin{aligned}
\mathrm{MSG}_{e} & =\left|\frac{\frac{g_{m} Z_{1}}{j \omega C_{\mu}}-Z_{1}-r_{\mathrm{ex}}\left(1+g_{m} Z_{1}\right)}{r_{\mathrm{ex}}\left(1+g_{m} Z_{1}\right)+Z_{1}}\right| \\
& =\left|\frac{\frac{g_{m} Z_{1}}{j \omega C_{\mu}}}{r_{\mathrm{ex}}\left(1+g_{m} Z_{1}\right)+Z_{1}}-1\right| .
\end{aligned}
$$

Under the assumption MSG»1 and $\left|g_{m} Z_{1}\right| \approx \beta \gg 1$ in the intermediate frequency range and using approximation $\alpha \approx 1$, $r_{e}=\alpha / g_{m} \approx 1 / g_{m}$ [24], (10) can be simplified as

$$
\begin{aligned}
\mathrm{MSG}_{e} & \approx\left|\frac{\frac{g_{m} Z_{1}}{j \omega C_{\mu}}}{r_{\mathrm{ex}}\left(1+g_{m} Z_{1}\right)+Z_{1}}\right| \\
& \approx\left|\frac{\frac{g_{m} Z_{1}}{j \omega C_{\mu}}}{r_{\mathrm{ex}} g_{m} Z_{1}+Z_{1}}\right|=\frac{1}{\omega C_{\mu}\left(r_{\mathrm{ex}}+r_{e}\right)} .
\end{aligned}
$$

For the CB configuration, since the value of $r_{o}$ is fairly large, (6) can thus be simplified as

$$
h_{r b}=\frac{j \omega r_{b} C_{\mu}}{1+j \omega C_{\mu}\left(r_{b}+r_{c}\right)} .
$$

From (7) and (12), MSG for the CB configuration is

$$
\mathrm{MSG}_{b}=\left|\frac{g_{m} Z_{2}+j \omega r_{b} C_{\mu}}{j \omega r_{b} C_{\mu}}\right|=\left|\frac{g_{m} Z_{2}}{j \omega r_{b} C_{\mu}}+1\right| .
$$

Since MSG $\gg 1$ and $\left|g_{m} Z_{2}\right| \approx \alpha$ in the intermediate frequency range, (13) can be further simplified as

$$
\mathrm{MSG}_{b}=\frac{\alpha}{\omega r_{b} C_{\mu}} .
$$

It is noted, from (11) and (14), that both $\mathrm{MSG}_{b}$ and $\mathrm{MSG}_{e}$ follow a $-10 \mathrm{~dB} / \mathrm{dec}$ degradation trend, which is commonly observed for MSG versus frequency. The ratio of MSG's between the $\mathrm{CB}$ and $\mathrm{CE}$ configurations is (using $r_{e}=\alpha / g_{m} \approx 1 / g_{m}$ [24] again)

$$
\frac{\mathrm{MSG}_{b}}{\mathrm{MSG}_{e}}=\frac{\alpha\left(r_{\mathrm{ex}}+\frac{1}{g_{m}}\right)}{r_{b}} \approx \frac{r_{\mathrm{ex}}+r_{e}}{r_{b}} .
$$

From (11) and (14), one can see that $\mathrm{MSG}_{e}$ is dependent on $r_{\mathrm{ex}}+r_{e}$ and $\mathrm{MSG}_{b}$ is dependent on $r_{b}$. For the CB configuration, $\mathrm{MSG}_{b}$ increases as $r_{b}$ decreases. Similarly, for the CE configuration, $\mathrm{MSG}_{e}$ is inversely proportional to $r_{\mathrm{ex}}+r_{e}$. This indicates that the parasitic resistance $r_{\mathrm{ex}}$ and $r_{b}$ should be reduced in order to improve $\mathrm{MSG}_{e}$ and $\mathrm{MSG}_{b}$, respectively. One can also notice that neither $\mathrm{MSG}_{e}$ nor $\mathrm{MSG}_{b}$ is dependent on the parasitic collector resistance $r_{c}$. Although increased $r_{c}$ can increase the RC delay (via $C_{b c} \cdot r_{c}$ ) of SiGe HBTs, which in turn reduces the device cut-off frequency $f_{T}$, there is no significant effect of $r_{c}$ on small-signal power gain within the frequency range of concern.

Considering that the potentially unstable frequency range of SiGe HBTs in the CE configuration may be different from the CB configuration, (15) should be valid in the shared unstable frequency range of the two configurations. Of more importance, it can be seen from (15) that the ratio, $\mathrm{MSG}_{b} / \mathrm{MSG}_{e}$ is determined by the relative values of $r_{\mathrm{ex}}+r_{e}$ and $r_{b}$. With a relatively large value of $r_{b}$ and a small value of $r_{\mathrm{ex}}+r_{e}$, the ratio $\mathrm{MSG}_{b} / \mathrm{MSG}_{e}$ can be smaller than unity. This case holds for industry mainstream SiGe HBTs. In these SiGe HBTs [Fig. 2(a)], the Ge mole fraction is graded across the base with a smaller (or zero) mole fraction at the emitter side and a larger mole fraction at the collector side in order to generate a quasi-electric field for assisting the transition of minority carrier across the neutral base region. Electron injection efficiency from emitter to base is not (at least not substantially) benefited from such a Ge profile. Nevertheless, the high electron injection efficiency from emitter to base is realized with a doping profile typical of Si BJTs, which has a high-to-low doping concentration from the emitter to the base and then to the collector region [Fig. 2(a)]. Because of the limited, low doping concentration (lower than the emitter region) in the base region, a relatively high base resistance $r_{b}$ is thus resulted. On the other hand, the values of parasitic emitter resistance $r_{\text {ex }}$ of these SiGe HBTs are always quite small owing to the thin, yet heavily doped poly Si emitter. Although the downscaling of emitter finger width of SiGe HBT can reduce the base resistance $r_{b}$ (to increase $f_{\max }$ ), it is important to point out that the relative values of $r_{b}$ and $r_{\text {ex }}$ cannot be changed by such scaling.

For power amplification using SiGe HBTs, an alternative Ge profile and thus an alternative doping profile can be employed. A large mole fraction of $\mathrm{Ge}$ at the emitter side in the base region can be used to create a significant bandgap offset (mainly in the valence band) between the emitter and the base region. The bandgap offset-enhanced injection efficiency can be traded for a very heavily doped base region [Fig. 2(b)]. Such a doping profile has always been employed in III-V HBTs. The high doping concentration in the base region can appreciably reduce base resistance $r_{b}$ such that the $f_{\max }\left(f_{\max }=\sqrt{f_{T} / 8 \pi r_{b} C_{\mu}}\right)$ can be enhanced. Moreover, the base resistance $r_{b}$ can thus be reduced to a very small value and becomes comparable to the parasitic emitter resistance $r_{\mathrm{ex}}$. In this case, the power gain ratio $\mathrm{MSG}_{b} / \mathrm{MSG}_{e}$ can be readily larger than unity.

In order to obtain a complete relationship between the $\mathrm{CE}$ and the CB configurations for any BJTs within the entire frequency range of interest, we further examine the power gain expressions of the two configurations in the high and the low frequency ranges. At very low and very high frequencies, BJTs are generally stable [25]. The (stable) power gain [also called maximum available power gain (MAG)] can be expressed as [25]

$$
G=\frac{\left|h_{f}\right|^{2}}{4 \operatorname{Re}\left(h_{i}\right) \operatorname{Re}\left(h_{o}\right)-2 \operatorname{Re}\left(h_{f} h_{r}\right)} .
$$

We now consider the MAG in these two frequency ranges. 


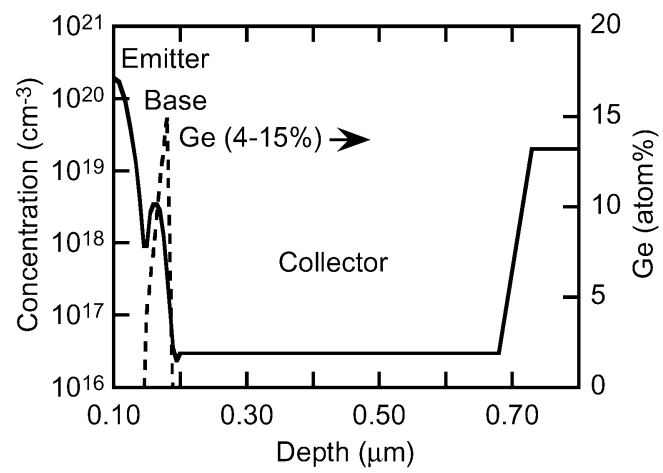

(a)

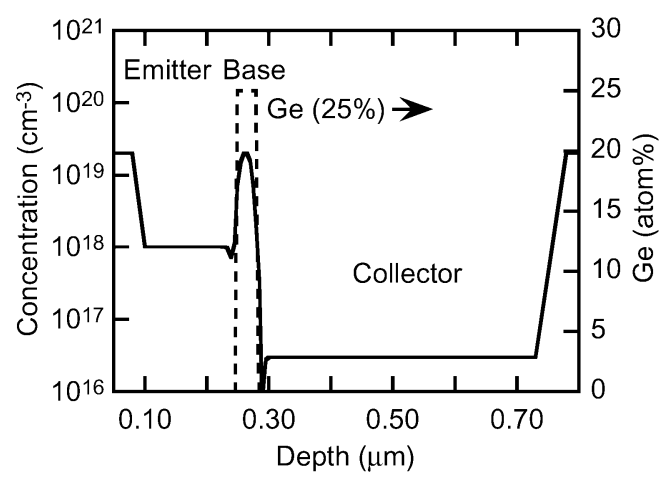

(b)

Fig. 2. Two representative doping profiles and Ge profiles of SiGe HBTs. (a) Doping profile typical of Si BJTs with graded Ge mole fraction (5-14\%) in the base region (peak base doping concentration, $3.5 \times 10^{18} \mathrm{~cm}^{-3}$ ). (b) Doping profile of SiGe HBTs with (box-type) high Ge mole fraction $(25 \%)$ present at the emitter-base junction and high base doping concentration $\left(2 \times 10^{19} \mathrm{~cm}^{-3}\right)$. Both structures have the same collector epi-layer structures.

\section{B. Low-Frequency Range}

In the low frequency range, The $H$-parameters (1)-(4) in the $\mathrm{CE}$ configuration can be simplified as the following:

$$
\begin{aligned}
& h_{i e}=r_{b}+r_{\pi}+\beta r_{\mathrm{ex}} \\
& h_{r e}=0 \\
& h_{f e}=\beta \\
& h_{o e}=\frac{1}{r_{o}}
\end{aligned}
$$

and the power gain $G_{e, l}$ (subscript l: low frequency) is expressed as

$$
G_{e, l}=\frac{r_{o} \beta^{2}}{4\left(r_{b}+r_{\pi}+\beta r_{\mathrm{ex}}\right)}
$$

Similarly, the $H$-parameters (5)-(8) in the CB configuration can be simplified as

$$
\begin{aligned}
h_{i b} & =\frac{\alpha\left(r_{b}+r_{\pi}\right)}{\beta}+r_{\mathrm{ex}} \\
h_{r b} & =\frac{\alpha\left(r_{b}+r_{\pi}\right)}{\beta r_{o}} \\
h_{f b} & =-\alpha \\
h_{o b} & =\frac{\alpha}{\beta r_{o}}
\end{aligned}
$$

and the power gain $G_{b, l}$ in this configuration, by assuming $\alpha \approx$ 1 and $\beta \gg 1$, is

$G_{b, l}=\frac{\alpha \beta r_{o}}{2 \alpha\left(r_{b}+r_{\pi}\right)+4 r_{\mathrm{ex}}+\frac{4 \alpha\left(r_{b}+r_{\pi}\right)}{\beta}} \approx \frac{\beta r_{o}}{2\left(r_{b}+r_{\pi}\right)+4 r_{\mathrm{ex}}}$

The power gain ratio (use $\beta r_{e}=r_{\pi}$ ) between the two configurations, thus, is

$$
\frac{G_{e, l}}{G_{b, l}} \approx \frac{\beta\left(r_{b}+r_{\pi}\right)+2 \beta r_{\mathrm{ex}}}{2\left(r_{b}+r_{\pi}\right)+2 \beta r_{\mathrm{ex}}}>1
$$

One can see, from (27), that in the low frequency range, the CE configuration always has a higher power gain than the $\mathrm{CB}$ configuration, regardless of the value of $r_{b}$. In addition, the higher is the current gain $(\beta)$ in the CE configuration, the higher is the power gain ratio (larger than unity) between the $\mathrm{CE}$ and the $\mathrm{CB}$ configurations.

\section{High-Frequency Range}

In the high-frequency range, the devices are unconditionally stable. MAG can be expressed as

$$
\operatorname{MAG}=\operatorname{MSG}\left(K-\sqrt{K^{2}-1}\right)
$$

where $K$ is the Rollett's stability factor (the $K$-factor) [26]

$$
K=\frac{2 \operatorname{Re}\left(Z_{11}\right) \operatorname{Re}\left(Z_{22}\right)-\operatorname{Re}\left(Z_{12} Z_{21}\right)}{\left|Z_{12} Z_{21}\right|}
$$

Due to the complication of the $K$-factor in MAG, a simplified expression of MAG for both $\mathrm{CE}$ and $\mathrm{CB}$ configurations is impossible to obtain. We thus take an alternative approach to compare $\mathrm{MAG}_{e}$ and $\mathrm{MAG}_{b}$.

After converting the $K$-factor into the $h$-parameter representation and substituting (1)-(4) and (5)-(8) with appropriate approximations, the $K$-factor for the $\mathrm{CE}$ and the $\mathrm{CB}$ configurations can be derived, respectively, as

$$
\begin{aligned}
& K_{\mathrm{CB}} \approx\left[\left(r_{b}+2 r_{\mathrm{ex}}\right) C_{\mu}+r_{e} C_{\pi}\right] \omega=k_{b} \omega \\
& K_{\mathrm{CE}} \approx\left[\left(r_{\mathrm{ex}}+2 r_{b}\right) C_{\mu}+r_{e} C_{\pi}\left(1+\frac{2 r_{b}}{r_{\mathrm{ex}}}\right)\right] \omega=k_{e} \omega
\end{aligned}
$$

These two analytical $K$-factor expressions are equivalent to the ones obtained by Tanaka et al. [23] when ignoring $r_{c}$. It can be easily shown [23] that $k_{e}>k_{b}$, indicating that the $K$-factor of the $\mathrm{CB}$ configuration increases with frequency at a smaller rate than the $\mathrm{CE}$ configuration. Consequently, the frequency point $\left(f_{K=1, \mathrm{CB}}\right)$ at which the $K$-factor of the CB configuration reaches unity (breakpoint of MSG/MAG) is larger than the corresponding frequency point of the $\mathrm{CE}$ configuration $\left(f_{K=1, \mathrm{CE}}\right)$, i.e., $f_{K=1, \mathrm{CB}}>f_{K=1, \mathrm{CE}}$. It is also because of $k_{e}>k_{b}$ that $\mathrm{MAG}_{e}$ and $\mathrm{MAG}_{b}$ (28) decrease with the frequency at different rates. By comparing the slopes of $\mathrm{MAG}_{e}$ and $\mathrm{MAG}_{b}$ versus frequency using $d\left(\mathrm{MAG}_{e}(\mathrm{~dB})\right) / d(\log \omega)$ and $d\left(\mathrm{MAG}_{b}(\mathrm{~dB})\right) / d(\log \omega)$, it can be shown that $\mathrm{MAG}_{b}$ decreases faster with frequency than $\mathrm{MAG}_{e}$. 


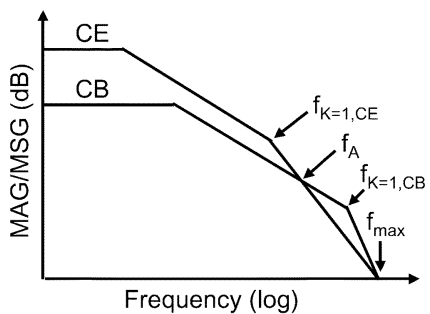

(a)

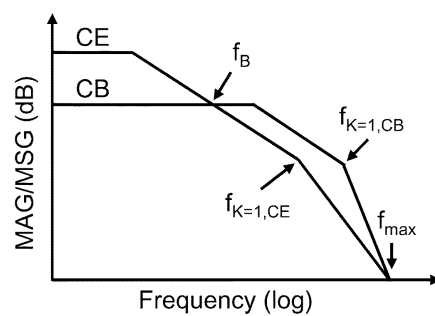

(b)
Fig. 3. Representative trends of $G_{\max }$ versus frequency for SiGe HBTs operated in the $\mathrm{CE}$ and the $\mathrm{CB}$ configurations, when (a) the base doping concentration is low, corresponding to Fig. 2(a) and (b) the base doping concentration is high, corresponding to Fig. 2(b).

If $\mathrm{MSG}_{b}>\mathrm{MSG}_{e}$ (15) for the case of $r_{b}<r_{e}+r_{\mathrm{ex}}$, in light of the fact of $f_{K=1, \mathrm{CB}}>f_{K=1, \mathrm{CE}}$, then it is always true that $\mathrm{MAG}_{b}>\mathrm{MAG}_{e}$. If $\mathrm{MSG}_{b}<\mathrm{MSG}_{e}$ (15) for the case of $r_{b}>$ $r_{e}+r_{\mathrm{ex}}$, the value of $k_{e} / k_{b}\left(=f_{K=1, \mathrm{CB}} / f_{K=1, \mathrm{CE}}\right.$, as seen from (30) and (31). It can be roughly approximated as $1+\left(2 r_{b} / r_{\mathrm{ex}}\right)$ [23] for simplicity) and that of $r_{b} /\left(r_{e}+r_{\mathrm{ex}}\right)$ [ $=\mathrm{MSG} / \mathrm{MSG}_{b}$, (15)] need to be compared in order to compare the relative size of $\mathrm{MAG}_{b}$ and $\mathrm{MAG}_{e}$. A straightforward comparison of these two ratios shows that $k_{e} / k_{b}>r_{b} /\left(r_{e}+r_{\text {ex }}\right)$ regardless of relative size of $r_{b}$ and $r_{e}+r_{\mathrm{ex}}$. When both MAG and frequency are plotted in logarithmic scale, $k_{e} / k_{b}>r_{b} /\left(r_{e}+r_{\mathrm{ex}}\right)$, in light of the fact that $\mathrm{MAG}_{b}$ and $\mathrm{MAG}_{e}$ merge together (with gain value of unity) at the same $f_{\max }[23]$, indicates that $\mathrm{MAG}_{b}$ must be larger than $\mathrm{MAG}_{e}$ in the frequency range of $f<f_{\max }$. As a result, in the high-frequency range, the $\mathrm{CB}$ configuration always offers higher power gain $\left(\mathrm{MAG}_{b}\right)$ than the $\mathrm{CE}$ configuration $\left(\mathrm{MAG}_{e}\right)$ in spite of the ratio of $\mathrm{MSG}_{b} / \mathrm{MSG}_{e}$.

Combining these analyses, the typical relationship of power gain $G_{\max }$, spanning the entire frequency range within $f_{\max }$, between the $\mathrm{CE}$ and the $\mathrm{CB}$ configurations for the two distinct doping profiles is sketched in Fig. 3. A conceptual insight can be used to further explain the different power gain characteristics in the $\mathrm{CE}$ and $\mathrm{CB}$ configurations. For the $\mathrm{CE}$ configuration, in the frequency range where both the current gain and voltage gain of a BJT are higher than unity, the power gain originates from the product of the current gain and the voltage gain. Otherwise, the power gain is originated either only from the current gain in the frequency range where $f_{T}>f_{\max }$, or only from the voltage gain where $f_{\max }>f_{T}$. In contrast, for the CB configuration, the power gain only originates from the voltage gain within the entire frequency range. At high operation frequencies, the current gain of the $\mathrm{CE}$ configuration degrades dramatically. Therefore, the $\mathrm{CB}$ configuration exhibits higher power gain than the $\mathrm{CE}$ configuration. At very low frequencies, because there is no current gain for the $\mathrm{CB}$ configuration, the $\mathrm{CE}$ configuration always offers higher power gain than the $\mathrm{CB}$ configuration. The higher is the current gain (or higher $f_{T}$ ) of the device, the wider is the frequency range where the CE configuration is favored for power amplification, leaving the frequency range where the CB offers higher power gain smaller. Furthermore, it can be expected that if $f_{T} \gg f_{\max }$ for a device, there will be no frequencies at which the $\mathrm{CB}$ configuration can be (or practically) used for power amplification. Conversely, if $f_{\max }>f_{T}$, the frequency range where the $\mathrm{CB}$ configuration is superior over $\mathrm{CE}$ configuration for power amplification will become larger. The relation of power gains between the $\mathrm{CE}$ and $\mathrm{CB}$ configurations, as depicted in Fig. 3, and the above descriptive synopsis can be applied to any types of bipolar junction transistors.

\section{Operation CONFIGURATION OF SiGe HBTs}

Based on the analyses in Section II, the appropriate operation configuration of SiGe HBTs is discussed here. For SiGe HBTs with low base doping concentrations [Fig. 2(a)], there is a frequency point, $f_{A}$, above which the power gain of $\mathrm{CB}$ configuration is higher than that of the CE configuration, as shown in Fig. 3(a). The frequency $f_{A}$ is the point where $\mathrm{MAG}_{e}$ and $\mathrm{MSG}_{b}$ converge. In order to find the value of $f_{A}$, we need to first obtain an analytical expression of $\mathrm{MAG}_{e}$. For this purpose, we use (16) to find $G_{e, h}$ (subscript h: high frequency).

The $H$-parameters in the CE configuration (1)-(4), in the high-frequency range, can be simplified as

$$
\begin{aligned}
h_{i e} & \approx r_{b}+\frac{1}{j \omega\left(C_{\pi}+C_{\mu}\right)} \\
h_{r e} & \approx \frac{C_{\mu}}{C_{\pi}+C_{\mu}} \\
h_{f e} & \approx \frac{g_{m}}{j \omega\left(C_{\pi}+C_{\mu}\right)}-\frac{C_{\mu}}{C_{\mu}+C_{\pi}} \\
h_{o e} & \approx \frac{C_{\mu}}{C_{\mu}+C_{\pi}}\left(g_{m}+j \omega C_{\pi}\right) .
\end{aligned}
$$

After substituting (32)-(35) into (16), $G_{e, h}$ can be expressed as

$$
G_{e, h}=\frac{\frac{g_{m}^{2}}{\omega^{2}\left(C_{\mu}+C_{\pi}\right)^{2}}+\frac{C_{\mu}^{2}}{\left(C_{\mu}+C_{\pi}\right)^{2}}}{4 r_{b} g_{m} \frac{C_{\mu}}{C_{\mu}+C_{\pi}}+2 \frac{C_{\mu}^{2}}{\left(C_{\mu}+C_{\pi}\right)^{2}}} .
$$

Since, usually, $C_{\mu} \ll C_{\pi}$, hence $\left.C_{\mu}^{2} /\left(C_{\mu}+C_{\pi}\right)^{2}\right)$ is very small compared to other terms. We thus can further simplify (36) as

$$
G_{e, h}=\frac{g_{m}}{4 \omega^{2} r_{b} C_{\mu}\left(C_{\mu}+C_{\pi}\right)} \approx \frac{g_{m}}{4 \omega^{2} r_{b} C_{\mu} C_{\pi}} .
$$

The frequency $f_{A}$ can then be obtained by equating (14) to (37). Since $C_{\mu} \ll C_{\pi}$, we can use approximation, $f_{T} \approx g_{m} /\left(2 \pi C_{\pi}\right)$ (from (34)). We thus obtain $f_{A}$ as

$$
f_{A}=\frac{f_{T}}{4 \alpha} \approx \frac{f_{T}}{4}
$$

where $f_{T}$ is the cutoff frequency of the CE configuration. Hence, for SiGe HBTs with a doping profile typical of Si BJTs, the CE configuration should be employed for power amplification within the frequency range between very low frequency and $\sim f_{T} / 4$. In addition, if $f_{A}$ is considerably smaller than $f_{\max }$ (e.g., in the case of $f_{T}<$ or $\approx f_{\max }$ ) there is still a wide frequency range (between $f_{A}$ and $f_{\max }$ ) in which the $\mathrm{CB}$ configuration offers higher power gain than the $\mathrm{CE}$ configuration. Consequently, the $\mathrm{CB}$ configuration may still be employed within a small frequency range beyond $f_{T} / 4$ depending on the actual power gain values. However, if the value of $f_{A}$ is too close to that of $f_{\max }\left(e . g\right.$., in the case $\left.f_{T}>f_{\max }\right)$, there will be no practically useful frequency range for $\mathrm{CB}$ operation. Instead, only the $\mathrm{CE}$ 


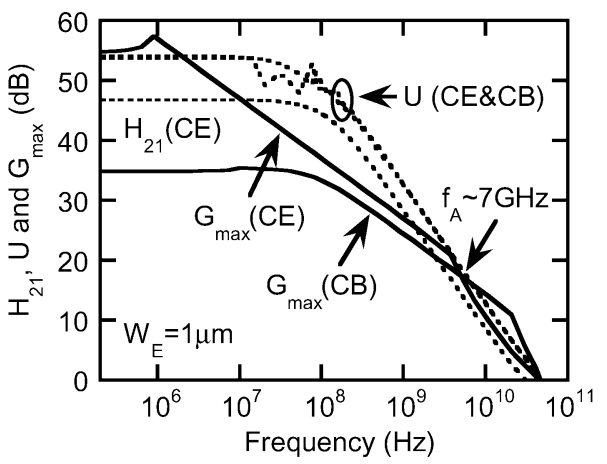

(a)

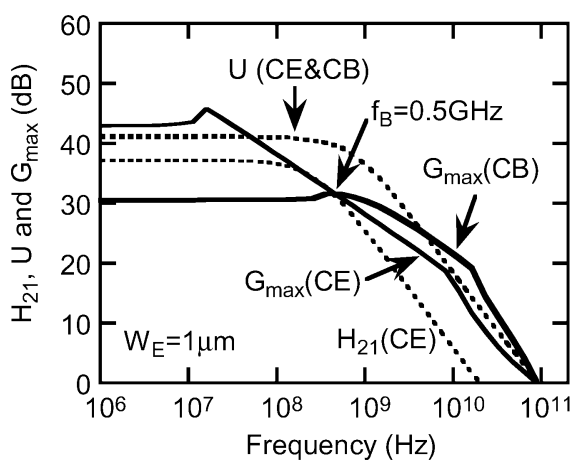

(b)

Fig. 4. Simulated power gain $\left(G_{\max }\right.$ and $\left.U\right)$ characteristics versus frequency for SiGe HBTs (a) with trapezoidal Ge profile and Si BJT doping profile, [shown in Fig. 2(a)] and, (b) with box-type Ge profile and high base doping concentration [shown in Fig. 2(b)]. The current gain characteristics versus frequency for these two SiGe HBTs are also shown for reference.

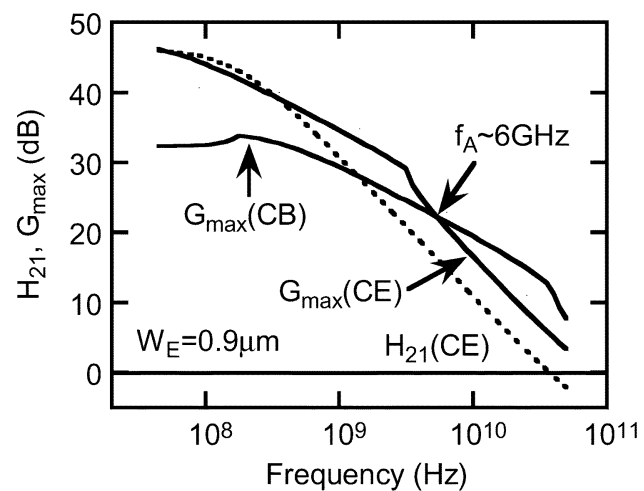

(a)

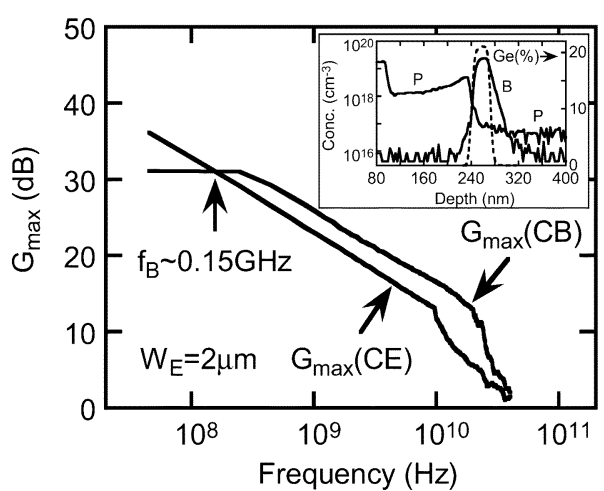

(b)

Fig. 5. Measured power gain versus frequency for SiGe HBTs (a) with a low base doping concentration $\left(\sim 10^{18} \mathrm{~cm}^{-3}\right)$ and (b) with a high base doping concentration $\left(2.5 \times 10^{19} \mathrm{~cm}^{-3}\right)$. The inset shows the SIMS results of the doping profile and Ge profile of the devices.

configuration should be favored over the entire frequency range of interest. Similarly, for SiGe HBTs with a heavily doped base region, as shown in Fig. 2(b), the power gain of the CB configuration is higher than that of the $\mathrm{CE}$ configuration beyond the frequency point $f_{B}$ (Fig. 3(b)). Since $f_{B}$ is the point where low-frequency $G_{b, l}$ meets $\mathrm{MSG}_{e}$, the value of $f_{B}$ can be obtained by equating (11) with (26) as

$$
f_{B} \approx \frac{r_{b}+r_{\pi}+2 r_{\mathrm{ex}}}{\pi C_{\mu} r_{o}\left(r_{\pi}+\beta r_{\mathrm{ex}}\right)}
$$

Due to the large value of $r_{o}, f_{B}$ is always far below the maximum oscillation frequency $f_{\max }$. Thus, for SiGe HBTs with a heavily doped base region when a high Ge mole fraction at the emitter-base junction is present, the $\mathrm{CB}$ configuration provides higher power gain than the $\mathrm{CE}$ configuration and thus should be employed for power amplification over a wide frequency range of interest (any frequency points beyond $f_{B}$ ). The best performance $\mathrm{SiGe}$ power HBTs operating at high frequencies $(X-$ to $K$-band) have been demonstrated by employing vertical structures with heavily doped based regions and using the CB configuration [9]-[11], [13].

\section{ANALYSES VERIFICATION}

In order to verify the power gain relationship between the $\mathrm{CE}$ and $\mathrm{CB}$ configurations within the entire frequency range of interest, 2-D simulations using MEDICI were performed on the two structures (emitter width $1 \mu \mathrm{m}$ ) shown in Fig. 2. The power gain $\left(G_{\max }\right)$ versus frequency of the SiGe HBTs shown in Fig. 2(a) and (b) is plotted in Fig. 4(a) and (b), respectively. These two curves clearly exhibit the power gain characteristics of SiGe HBTs as afore discussed (Fig. 3). The unilateral power gain $(U)$ and current gain $\left(H_{21}\right)$ are also shown for reference. As one can expect, there is no difference on $U$ between the two configurations and the same $f_{\max }$ value is reached by $U$ and $G_{\max }$ for both configurations. This further proves that, while $U$ can be used for calculating $f_{\max }$ and for comparing RF performance of devices under the same configuration, it does not contain informative power gain values for circuit design. Since the simulated device structures are free of interconnect parasitics, the simulation results should be considered as the typical characteristics of intrinsic devices. Furthermore, the simulated power gain values and the relationship between the $\mathrm{CE}$ and the $\mathrm{CB}$ configurations quantitatively agree with the theoretical analysis.

We also measured $S$-parameters from a commercial power SiGe HBT (emitter width $0.9 \mu \mathrm{m}$ ) that has a lower doping concentration $\left(\sim 10^{18} \mathrm{~cm}^{-3}\right)$ in the base than in the emitter region. The small-signal equivalent circuit parameters were then extracted from, and well fitted to, the measured $S$-parameters. The power gain $G_{\max }$ was calculated for both the $\mathrm{CE}$ and the $\mathrm{CB}$ configurations using the equivalent circuit parameters and plotted versus frequency as shown in Fig. 5(a). One can see that 
$\mathrm{MSG}_{e}$ is higher than $\mathrm{MSG}_{b}$ between the lowest measured frequency and $\sim 6 \mathrm{GHz}\left(f_{A}\right)$. Good agreement between theory (38) and measurement was achieved. Beyond $f_{A}=6 \mathrm{GHz}$, the power gain values are still useful for power amplification using this device. As a result, the CB instead of the CE configuration may be employed judiciously for power amplification in this high-frequency range, while the CE configuration should be used in the range of below $\sim 6 \mathrm{GHz}$.

For comparison, the $\mathrm{CE}$ and the $\mathrm{CB}$ power SiGe HBTs with high base doping concentrations $\left(2.5 \times 10^{19} \mathrm{~cm}^{-3}\right.$, see the inset for SIMS profile) were also measured and the $G_{\max }$ was plotted versus frequency in Fig. 5(b). As expected, $\mathrm{MSG}_{b}$ and $\mathrm{MAG}_{b}$ are larger than $\mathrm{MSG}_{e}$ and $\mathrm{MAG}_{e}$, respectively, in the frequency range of from $0.15 \mathrm{GHz}\left(f_{B}\right)$ to $40 \mathrm{GHz}\left(\sim f_{\max }\right)$. In addition, the power gain ratios quantitatively agree with predicted values in both the stable and the unstable frequency regions. Thus, the CB instead of the CE configuration should be used for power amplification using this type of SiGe HBTs over a wide frequency range (beyond $f_{A}=150 \mathrm{MHz}$ ). Again, one can observe that, at very low frequencies (close to DC, not useful for RF amplification though), the SiGe HBTs have higher power gain in the $\mathrm{CE}$ than in the $\mathrm{CB}$ configuration regardless of the high base doping concentration employed in the devices (27). It should be pointed out that, although two representative Ge and doping profiles [Fig. 2(a) and (b)] are used to illustrate the differences of power gain characteristics between the $\mathrm{CE}$ and the $\mathrm{CB}$ configurations, the selection of proper operation configuration for SiGe HBTs is mainly determined by the actual device parameters. For example, if the total base resistance of SiGe HBTs shown in Fig. 2(a) can be reduced (e.g., by increasing base doping concentration and employing a box-like trapezoidal Ge profile), $\mathrm{MSG}_{b}$ can be made close to, equal [a situation in between Fig. 3(a) and (b)] or even higher than $\mathrm{MSG}_{e}$. In such "transition" cases, even in the intermediate frequency range, the proper operation (not necessarily the $\mathrm{CE}$ ) configuration should thus be explored in order to maximize the power gain values of $\mathrm{SiGe}$ HBTs. Furthermore, for power amplification using SiGe HBTs and considering the option of operation configurations, the heterostructures can be optimized merely for a high $f_{\max }$. This is different from high-speed SiGe HBTs for which simultaneous high $f_{T}$ and $f_{\max }$ or just a high $f_{T}$ is required.

\section{CONCLUSION}

The difference of power gain characteristics of BJTs between the $\mathrm{CE}$ and the $\mathrm{CB}$ configurations has been analytically explained and verified with 2-D MEDICI simulation as well as measurement. The implication of the analyses on the operation configurations of SiGe HBTs has been studied. Depending on the device heterostructure, operation frequency, stability condition, and the relation between $f_{T}$ and $f_{\max }$, the CE and the $\mathrm{CB}$ configurations should be judiciously employed in order to maximize the power gain values of SiGe HBTs when employing them for RF and microwave power amplification. Specific operation frequency ranges have been given for guiding the selection of operation configurations.

\section{REFERENCES}

[1] U. Erben, M. Wahl, A. Schüppen, and H. Schumacher, "Class-A SiGe HBT power amplifiers at $C$-band frequencies," IEEE Microw. Guided Wave Lett., vol. 5, no. 12, pp. 435-436, Dec. 1995.

[2] A. Schüppen, S. Gerlach, H. Dietrich, D. Wandrei, U. Seiler, and U. König, "1-W SiGe power HBT's for mobile communication," IEEE Microw. Guided Wave Lett., vol. 6, no. 9, pp. 341-343, Sep. 1996.

[3] D. R. Greenberg, M. Rivier, P. Girard, E. Bergeault, J. Moniz, D. Ahlgren, G. Freeman, S. Subbanna, S. J. Jeng, K. Stein, D. Nguyen-Ngoc, K. Schonenberg, J. Malinowski, D. Colavito, D. L. Harame, and B. Meyerson, "Large-signal performance of high-BVCEO graded epi-base SiGe HBTs at wireless frequencies," in IEDM Tech. Dig., Dec. 1997, pp. 799-802.

[4] J. N. Burghartz, J. Plouchart, K. A. Jenkins, C. S. Webster, and M. Soyuer, "SiGe power HBTs for low-voltage, high-performance RF applications," IEEE Electron Device Lett., vol. 19, no. 4, pp. 103-105, Apr. 1998.

[5] J. D. Cressler, "SiGe HBT technology: A new contender for Si-based RF and microwave circuit applications," IEEE Trans. Microw. Theory Tech., vol. 46, no. 5, pp. 572-589, May 1998.

[6] R. Götzfried, F. Beißwanger, S. Gerlach, A. Schüppen, H. Dietrich, U. Seiler, K.-H. Bach, and J. Albers, "RFICs for mobile communication systems using SiGe bipolar technology," IEEE Trans. Microw. Theory Tech., vol. 46, no. 5, pp. 661-668, May 1998.

[7] R. Götzfried, F. Beißwanger, and S. Gerlach, "Design of RF integrated circuits using SiGe bipolar technology," IEEE J. Solid-State Circuits, vol. 33, no. 9, pp. 1417-1422, Sep. 1998.

[8] P.-D. Tseng, L. Zhang, G.-B. Gao, and M. F. Chang, "A 3-V monolithic SiGe HBT power amplifier for dual-mode (CDMA/AMPS) cellular handset applications," IEEE J. Solid-State Circuits, vol. 35, no. 9, pp. 1338-1344, Sep. 2000.

[9] Z. Ma, S. Mohammadi, P. Bhattacharya, L. P. B. Katehi, S. A. Alterovitz, and G. E. Ponchak, "High power $X$-band $(8.4 \mathrm{GHz}) \mathrm{SiGe} / \mathrm{Si}$ heterojunction bipolar transistor," Electron. Lett., vol. 37, pp. 790-791, Jun. 2001.

[10] Z. Ma, S. Mohammadi, L. Lu, P. Bhattacharya, L. P. B. Katehi, S. A. Alterovitz, and G. E. Ponchak, "An $X$-band high-power amplifier using SiGe-Si HBT and lumped passive components," IEEE Microw. Wireless Components Lett., vol. 11, no. 7, pp. 287-289, Jul. 2001.

[11] Z. Ma, S. Mohammadi, P. Bhattacharya, L. P. B. Katehi, S. A. Alterovitz, G. E. Ponchak, K. M. Strohm, and J.-F. Luy, "Ku-band (12.6 GHz) $\mathrm{SiGe/Si} \mathrm{high-power} \mathrm{heterojunction} \mathrm{bipolar} \mathrm{transistors,"} \mathrm{Electron.} \mathrm{Lett.,}$ vol. 37, pp. 1140-1142, Aug. 2001.

[12] D. L. Harame, D. C. Ahlgren, D. D. Coolbaugh, J. S. Dunn, G. G. Freeman, J. D. Gillis, R. A. Groves, G. N. Hendersen, R. A. Johnson, A. J. Joseph, S. Subbanna, A. M. Victor, K. M. Watson, C. S. Webster, and P. J. Zampardi, "Current status and future trends of SiGe BiCMOS technology," IEEE Trans. Electron Devices, vol. 48, no. 11, pp. 2575-2594, Nov. 2001.

[13] Z. Ma, S. Mohammadi, P. Bhattacharya, L. P. B. Katehi, S. A. Alterovitz, and G. E. Ponchak, "A high-power and high-gain $X$-band $\mathrm{Si} / \mathrm{SiGe} / \mathrm{Si}$ heterojunction bipolar transistor," IEEE Trans. Microw. Theory Tech., vol. 50, no. 4, pp. 1101-1108, Apr. 2002.

[14] M. A. Margarit, D. Shih, P. J. Sullivan, and F. Ortega, "A 5-GHz BiCMOS RFIC front-end for IEEE 802.11a/hiperLAN wireless LAN," IEEE J. Solid-State Circuits, vol. 38, no. 7, pp. 1284-1287, Jul. 2003.

[15] A. Keerti and A. Pham, "SiGe power devices for 802.11a wireless LAN applications at $5 \mathrm{GHz}$," Electron. Lett., vol. 39, pp. 1218-1220, Aug. 2003.

[16] A. J. Joseph, J. Dunn, G. Freeman, D. L. Harame, D. Coolbaugh, R. Groves, K. J. Stein, R. Volant, S. Subbanna, V. S. Marangos, S. S. Onge, E. Eshun, P. Cooper, J. B. Johnson, J.-S. Rieh, B. Jagannathan, V. Ramachandran, D. Ahlgren, D. Wang, and X. Wang, "Product applications and technology directions with SiGe BiCMOS," IEEE J. Solid-State Circuits, vol. 38, no. 9, pp. 1471-1478, Sep. 2003.

[17] W. Bakalski, W. Simbürger, R. Thüringer, A. Vasylyev, and A. Scholtz, "A fully integrated 5.3-GHz 2.4-V 0.3-W SiGe bipolar power amplifier with 50- $\Omega$ output," IEEE J. Solid-State Circuits, vol. 39, no. 7, pp. 1006-1014, Jul. 2004.

[18] J.-S. Rieh, B. Jagannathan, H. Chen, K. Schonenberg, S.-J. Jeng, M. Khater, D. Ahlgren, G. Freeman, and S. Subbanna, "Performance and design consideration for high speed SiGe HBTs of $f_{T} / f_{\max }=375 \mathrm{GHz} / 210 \mathrm{GHz}$," in Proc. Int. Conf. Indium Phosphide Related Materials, May 2003, pp. 374-377. 
[19] M. Racanelli, P. Ma, and P. Kempf, "SiGe BiCMOS technology for highly integrated wireless transceivers," in Gallium Arsenide Integrated Circuits Tech. Dig., Nov. 2003, pp. 183-186.

[20] N. L. Wang, N. H. Sheng, M. F. Chang, W. J. Ho, G. J. Sullivan, E. A. Sovero, J. A. Higgins, and P. M. Asbeck, "Ultrahigh power efficiency operation of common-emitter and common-base HBTs at $10 \mathrm{GHz}, "$ IEEE Trans. Microw. Theory and Tech., vol. 38, no. 10, pp. 1381-1390, Oct. 1990.

[21] P. M. Asbeck, F. M.-C. Chang, K.-C. Wang, G. J. Sullivan, and D. T. Cheung, "GaAs-based heterojunction bipolar transistors for very high performance electronic circuits," Proc. IEEE, vol. 81, no. 12, pp. 1709-1726, Dec. 1993.

[22] M. J. W. Rodwell, M. Urteaga, T. Mathew, D. Scott, D. Mensa, Q. Lee, J. Guthrie, Y. Betser, S. C. Martin, R. P. Smith, S. Jaganathan, S. Krishnan, S. I. Long, R. Pullela, B. Agarwal, U. Bhattacharya, L. Samoska, and M. Dahlstrom, "Submicron scaling of HBTs," IEEE Trans. Electron Devices, vol. 48, no. 11, pp. 2606-2624, Nov. 2001.

[23] S. Tanaka, Y. Amamiya, S. Murakami, H. Shimawaki, N. Goto, Y. Takayama, and K. Honjo, "Design considerations for millimeter-wave power HBT's based on gain performance analysis," IEEE Trans. Electron Devices, vol. 45, no. 1, pp. 36-44, Jan. 1998.

[24] P. R. Gray, P. J. Hurst, S. H. Lewis, and R. G. Meyer, Analysis and Design of Analog Integrated Circuits, 4th ed. New York: Wiley, 2000, pp. 183-184.

[25] R. L. Prichard, Electrical Characteristics of Transistors. New York: McGraw-Hill, 1967, ch. 5 \& 8.

[26] J. M. Rollett, "Stability and power-gain invariants of linear two ports," IRE Trans. Circuit Theory, vol. CT-9, pp. 29-32, 1962.

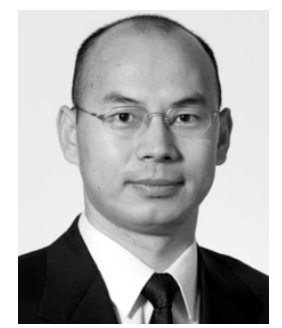

Zhenqiang Ma (S'98-M'01) received the B.S degree in applied physics and the B.E. degree in electrical engineering from Tsinghua University, Beijing, China, in 1991. He received the M.S degree in nuclear science, and the M.S.E. and Ph.D. degrees, both in electrical engineering, from the University of Michigan, Ann Arbor, in 1997 and 2001, respectively. His doctoral research focuses on the development SiGe power HBTs for high-frequency microwave applications.

In June 2001, he joined the R\&D team of Conexant Systems and later its spinoff Jazz Semiconductor, Newport Beach, CA, where he worked on the development, characterization, and modeling of high-speed SiGe HBTs. In August 2002, he left Jazz to join the faculty of the Department of Electrical and Computer Engineering, University of Wisconsin, Madison, as an Assistant Professor. His research interests include SiGe and III-V HBT/FET devices and technologies, RF circuits, MMICs, reliability of devices and circuits, $\mathrm{Si}$ and $\mathrm{SiGe}$ strained materials and physics, novel integration scheme, wide bandgap semiconductor materials, devices and applications, and nanoscale devices. He has published over 30 peer-reviewed technical papers related to his research. He was featured in the Massachusetts Institute of Technology Technology Review magazine for his innovation on cellphone power devices in 2004

Dr. Ma served as a Technical Reviewer of IEEE TRANSACTIONS ON Electron Devices, IEeE TRANSACtions on Circuits AND Systems II. He served as a Technical Reviewer of Elsevier Superlattices and Microstructures, Semiconductor Science and Technology, Electrochemical and Solid-State Letters, Journal of Physics D: Applied Physics, and the ETRI Journal

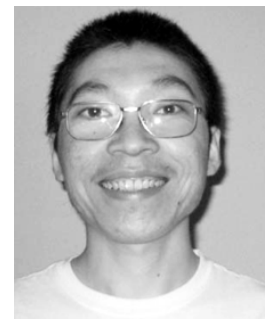

Ningyue Jiang (S'04) received the B.S. degree in electronic engineering from Tsinghua University, Beijing, China, in 2000, the M.S. degree in electrical engineering from the University of New Mexico, Albuquerque, NM, in 2002, and is currently pursuing the Ph.D. degree in electrical engineering at the University of Wisconsin, Madison.

His research interests include SiGe HBT devices and circuits for RF and microwave applications. 